\title{
ANALYSIS AND TOPOLOGY OF HYPERPLANE COMPLEMENTS: THE GENERALIZED WITT FORMULA
}

\author{
By Michel JAMBU
}

\section{Introduction.}

The classical Witt formula which gives the dimensions of the homogeneous components of the free Lie algebra over a finite set, has a nice interpretation as a relation between the topology, i. e. cohomology and homotopy of the complement of a finite set of $\boldsymbol{C}$, and the analysis, i.e. an ordinary linear differential equation with regular singular points at this finite set of $\boldsymbol{C}$.

Such a relation remains true for complements of some hyperplane arrangements such as complexified Coxeter arrangements and fiber-type arrangements.

Namely, let $\mathcal{A}$ be a finite family of hyperplanes of $\boldsymbol{C}^{n}$ through the origin and let $M=C^{n} \backslash \bigcup_{H \in A} H$ be the complement. The cohomology algebra $H^{*}(M ; K)$, where $K=\boldsymbol{Z}, \boldsymbol{Q}, \boldsymbol{R}$ or $\boldsymbol{C}$ is isomorphic to $\mathcal{E} / I$ where $\mathcal{E}$ is the free exterior algebra over $A$ and $I$ is the ideal defined by some dependence relations between the hyperplanes of $\mathcal{A}$. Moreover:

$$
P_{M}(t)=\Sigma_{p \geqq 0}\left(\operatorname{rank} H^{p}(M)\right) t^{p}=\Sigma_{x \in L(A)} \mu(x)(-t)^{\operatorname{codim} \mathrm{x}}
$$

where $L(\mathcal{A})$ is the lattice of intersections hyperplanes ordered by reverse inclusion, $\mu(x)=\mu(0, x), \mu$ being the Möbius function. These results are due to $\mathrm{P}$. Orlik and L. Solomon [OS].

The algebra of the integrable logarithmic connections along $\mathcal{A}$ is called the holonomy Lie algebra of $M$ and is denoted $\mathcal{G}_{M}$. T. Kohno [K1] showed that $\mathcal{G}_{M}=\operatorname{Lib}(A) / \mathscr{I}$ where $|A|=|\mathcal{A}|$ and $\mathscr{\Omega}$ is the ideal defined by some dependence relations between the hyperplanes of $A$.

Let $\mathcal{L}_{M}$ be the Malcev algebra of $M$ which is obtained (cf Sullivan [S]) from the 1-minimal model of $M$. Using the mixed Hodge structure on the minimal model, T. Kohno [K2] showed that:

$$
\mathcal{G}_{M}^{*} \approx \mathcal{L}_{M}
$$

where $\mathcal{G}_{M}^{*}$ is the nilpotent completion of $\mathcal{G}_{M}$.

Then $\mathrm{T}$. Kohno [K3] proved that:

$$
\varphi_{\jmath}(M)=\operatorname{dim}\left(\Gamma_{j} \mathcal{G}_{M} / \Gamma_{j+1} \mathcal{G}_{M}\right)=\operatorname{rank}\left(\Gamma_{j} \pi_{1}(M) / \Gamma_{\jmath+1} \pi_{1}(M)\right)
$$

Received February 8, 1991. 
and

$$
\sum_{p \geqq 0} \chi(p) t^{p}=\Pi_{\jmath \geqq 1}\left(1-t^{\jmath}\right)^{-\varphi_{J-1}(M)}
$$

where $\chi(p)=\operatorname{dim} \mathcal{E}_{p}\left(\mathcal{Q}_{M}\right)$, the dimension of the $p$ th homogeneous component of the universal algebra of $\mathcal{G}_{M}$.

If $\mathcal{A}$ is a complexified Coxeter arrangement or a fiber-type arrangement, for instance, then the following relation, called LCS formula is satisfied ([FR1], $[\mathrm{K} 4],[\mathrm{J}])$ :

$$
\sum_{p \geqq 0} \chi(p) t^{p}=\Pi_{\jmath \geq 1}\left(1-t^{j}\right)^{-\varphi_{J-1}(M)}=\left(P_{M}(-t)\right)^{-1}
$$

In this paper, we begin explaining how the LCS formula is a generalized Witt formula. However, if for the complexified Coxeter arrangements and the fiber-type arrangements, there are several methods to prove such a formula, M. Falk and R. Randell [FR2] noticed that for an arbitrary arrangement " $\cdots$ the LCS formula is virtually impossible to verify ...". Hence in the last section, following a suggestion of T. Kohno, we develop some method which can be useful to verify the $L C S$ formula.

According to $\mathrm{K}$. Aomoto [Ao], we consider the complex $(R ., \partial$.$) defined as$ follows :

$$
\begin{gathered}
R_{k}=\operatorname{Hom}_{\mathcal{E}\left(\underline{G}_{M}\right)}\left(\mathcal{E}\left(\underline{G}_{M}\right) \otimes_{\boldsymbol{Q}} H^{k}(M ; \boldsymbol{Q}), \mathcal{E}\left(\underline{G}_{M}\right)\right) \\
\partial_{k}(f)(x \otimes \varphi)=f\left(x \sum_{H \in A} X_{H} \otimes(H \cup \varphi)\right)
\end{gathered}
$$

where $X_{H}$ is the element of the set $A$ defining $\mathcal{G}_{M}$ which corresponds to $H \in \mathcal{A}$ and $\varphi \in H^{k-1}(M ; \boldsymbol{Q})$ and $\mathcal{E}\left(\mathscr{G}_{M}\right)$ denotes the universal enveloping algebra of $\mathcal{G}_{M}$.

If $H_{j}(R)=$.0 for any $j>0$, i. e. the complex is acyclic, then the LCS formula is satisfied. In order to prove the acyclicity of this complex, we introduce a structure of graded algebra on $H^{*}(M)$ in such a way that the spectral sequence of the associated filtered complex satisfies:

$$
E_{1}{ }^{p q}=0 \text { if } p+q \neq 0
$$

As an example, we construct such a filtration for the fiber-type arrangements.

The author wants to express his deep gratitude to Mutsuo Oka for his interest in this work and would like to thank Tokyo Institute of Technology and the organizers of the Workshop on Singularities in August 1990 for their hospitality.

Remark. Throughout the sections I to III we assume, for simplicity, $K=C$ although the results can be extended to $\boldsymbol{Q}$ or $\boldsymbol{C}$.

\section{Classical Witt formula.}

I. 1. Let $A=\left\{X_{1}, \cdots, X_{l}\right\}$ and let $\operatorname{Lib}(A)=\bigoplus_{n \geq 1} \operatorname{Lib}_{n}(A)$ be the fre Lie algebra. 
The dimensions of the homogeneous component of $L i b(A)$ are given by the classical Witt formula:

$$
\operatorname{dim} \operatorname{Lib}_{n}(A):=N_{n}=n^{-1} \sum_{d \mid n} \mu(d) l^{n d^{-1}}
$$

where $\mu$ is the classıcal Möbius function given by:

$$
\begin{aligned}
& \mu: N^{*} \longrightarrow\{-1,0,1\} \\
& n \rightsquigarrow 0 \text { if } p^{2} \mid n \text { where } p \text { is prime } \\
& n \rightsquigarrow(-1)^{k} \text { if } n=p_{1} \cdots p_{k}, p_{\imath} \neq p_{3}
\end{aligned}
$$

The enveloping algebra $\mathcal{E}(\operatorname{Lib}(A))$ is the free associative algebra $K\langle A\rangle$ and the canonical morphism

$$
\operatorname{Lib}(A) \longrightarrow K\langle A)
$$

is injective. Moreover, there exists a sequence $\left\{z_{1}, z_{2}, \cdots\right\}$ of homogeneous Lie elements with nodecreasing degrees such that:

$\left\{z_{1}, z_{2}, \cdots\right\}$ is a base of the space of the Lie elements

$\left\{z^{e_{1}}{ }_{i 1} \cdots z^{e_{k}}{ }_{i k}, 1 \leqq \imath_{1}<\cdots<\imath_{k}, k \geqq 1, e_{1}, \cdots, e_{k} \in N\right\} \cup\{1\}$ is a base of $K\langle A\rangle$.

Then $\left\{z_{1}, \cdots, z_{N_{1}}\right\}$ are the degree 1 elements, $\left\{z_{N_{1}+1}, \cdots, z_{N_{1}+N_{2}}\right\}$ are the degree 2 elements $\cdots$. The number of possibilities of selecting $n$ objects (repetitions allowed) out of a set of $N$ different ones equals the coefficient of $t^{n}$ in the power series expansion $(1-t)^{-N}$. On the other hand, $\operatorname{dim} K_{n}\langle A\rangle=l^{n}$, then :

$$
\Pi_{p \geqq 1}\left(1-t^{\jmath}\right)^{-N} \jmath=\sum_{n \geqq 0} l^{n} t^{n}=(1-l t)^{-1}
$$

Taking logarithms, differentiating with respect to $t$, after multiplication by $t$ and by application of the Möbius inversion, we obtain the classical Witt formula. Henceforth, in the following we call the relation $\left(^{*}\right)$ the classical Witt formula.

Remark. Let $F_{l}$ be the free group on $l$ generators $\alpha_{1}, \cdots, \alpha_{l}$ and let $\left(\Gamma_{n} F_{l}\right)_{n \in N}$ be the lower central series. Then there exist natural isomorphisms as abelian groups :

$$
\operatorname{Lib}_{n}(A) \longrightarrow \Gamma_{n} F_{l} / I^{\prime}{ }_{n+1} F_{l}
$$

\section{2. Topological interpretation of the classical Witt formula.}

Let $M=C \backslash\left\{\alpha_{1}, \cdots, \alpha_{l}\right\}$, then :

$$
\begin{aligned}
& \pi_{1}(M ; *) \approx F_{l}, \quad \text { then } N_{\jmath}=\operatorname{rank}\left(\Gamma_{j} \pi_{1}(M ; *) / \Gamma_{\jmath+1} \pi_{1}\left(M *^{*}\right)\right) \\
& H^{0}(M ; \boldsymbol{Z}) \approx \boldsymbol{Z}, \quad H^{1}(M ; \boldsymbol{Z}) \approx \boldsymbol{Z}^{l} \text { and } H^{i}(M ; \boldsymbol{Z})=\{0\} \quad \text { for } i>1
\end{aligned}
$$

and the Poincaré polynomial of $M$ is:

$$
P_{M}(t)=1+l t
$$


The classical Witt formula $\left(^{*}\right)$ establishes a relation between the fundamental group and the cohomology of $M$ :

$$
\Pi_{\jmath \geqq 1}\left(1-t^{\jmath}\right)^{-N_{\jmath}}=(1-l t)^{-1}=\left(P_{M}(-t)\right)^{-1}
$$

The second term of the Witt formula $(*), \sum_{n \geq 0} l^{n} t^{n}$, is the Poincaré series of the enveloping algebra $K\langle A\rangle$ of the free Lie algebra $\operatorname{Lib}(A)$.

Consider the first order linear differential equation:

$$
d Y=\omega Y
$$

where $\omega=\left(\omega^{\imath j}\right)_{1 \leqq \imath, \jmath \leqq m}$ and $\omega^{\imath \jmath}=\sum_{k-1}^{l} a^{\imath \jmath}{ }_{k} d \log \left(t-\alpha_{k}\right), a^{\imath \jmath}{ }_{k} \in C$. $\omega$ is a meromorphic $g l(m ; C)$-valued 1 -form on $M$ and defines a meromorphic connection $\nabla$ on the trivial bundle $\boldsymbol{C}^{m} \times M \rightarrow M$ by :

$$
\nabla f=d f-f \omega
$$

where $f: M \rightarrow C^{m}$ is a locally defined function. This connection is holomorphic on $C$ and has regular singular points at $\left\{\alpha_{1}, \cdots, \alpha_{l}\right\}$.

The transport function:

$$
T: P M \longrightarrow G l(m ; C)
$$

where $P M$ denotes the space of piecewise smooth maps $\gamma:[0,1] \rightarrow M$ is defined as follows : let $\gamma_{t}(s)=\gamma(s t)$, then $T(\gamma)$ is the solution at $t=1$ of the equation:

$$
d T\left(\gamma_{t}\right)=T\left(\gamma_{t}\right) \gamma^{*} \omega, \quad T\left(\gamma_{0}\right)=1
$$

An explicit formula for $T$ is given in terms of $\omega$ by Picard iteration along $\gamma$ where $\int \omega \omega \cdots \omega$ are iterated integrals $[\mathrm{Ch}]$ :

$$
T(\gamma)=I+\int_{\gamma} \omega+\int_{\gamma} \omega \omega+\cdots
$$

Moreover $\omega$ is integrable, i. e. $\omega \wedge \omega=d \omega=0$, then the value of $T$ on the path $\gamma$ depends only on its homotopy class relative to its endpoints.

Thus, $T$ induces the monodromy representation:

$$
\begin{aligned}
& \rho: \pi_{1}(M ; *) \longrightarrow G l(m ; C) \\
& \gamma \leftrightarrow \rightarrow I+\int_{\gamma} \omega+\int_{\gamma} \omega \omega+\cdots
\end{aligned}
$$

Notice that the series converges absolutly.

Examples. 1) $M=C \backslash\{0\}$ and $d Y=P z^{-1} d z Y, P \in M_{m}(\boldsymbol{C})$. Let $\gamma:[0,1]-\rightarrow M$ where $\gamma(t)=\exp (2 i \pi t)$. Then $\gamma^{*} \omega=2 i \pi P d t$ where $\omega=P z^{-1} d z, \int_{\gamma} \overbrace{\omega \cdots \omega}^{r \text { times }}=(2 i \pi P)^{r} / r$ ! and $\rho(\gamma)=\exp (2 i \pi P)$. 
2) $M=C \backslash\{0,1\}$ and $d Y=\omega Y$ where

$$
\omega=\left(\begin{array}{ccc}
0 & z^{-1} d z & 0 \\
0 & 0 & (1-z)^{-1} d z \\
0 & 0 & 0
\end{array}\right)
$$

Let $\Omega=C \backslash\{]-\infty, 0] \cup[1,+\infty[\}$. The matrix

$$
u(z)=\left(\begin{array}{ccc}
1 & \log z & \operatorname{dilog} z \\
0 & 1 & \log (1 / 1-z) \\
0 & 0 & 0
\end{array}\right)
$$

satisfies the equation $d u=\omega u$ on $\Omega$, where we take the principal determination of the logarithms and dilogz is the analytic continuation of the series $\Sigma_{n \geqq 1} t^{n} / n^{2}$ which converges for $|t| \leqq 1$.

Let $M=C \backslash\left\{\alpha_{1}, \cdots, \alpha_{l}\right\}$ and $d Y=\omega Y$ as above. We can express $\omega$ in terms of $\omega^{k}=d \log \left(t-\alpha_{k}\right), \quad k=1, \cdots, l$

$$
\omega=\sum_{k=1}^{l} \omega^{k} A^{k}
$$

where each $A^{k}$ is a constant matrix.

Let $A=\left\{X_{1}, \cdots, X_{l}\right\}$ and the homomorpeism :

$$
\begin{gathered}
\theta: \pi_{1}(M ; *) \longrightarrow C\langle\langle A\rangle\rangle \\
\gamma \rightsquigarrow \sum_{1 \leq i_{1} \leq \cdots \leqq \imath_{k} \leq l} \int_{\gamma} \omega^{\imath_{1}} \cdots \omega^{\imath_{k}} X_{\imath_{1}} \cdots X_{\imath_{k}}
\end{gathered}
$$

The monodromy representation $\rho$ is obtained by substituting $A^{\imath \jmath} \in g l(m ; \boldsymbol{C})$ to $X_{\imath \jmath}$. Finally, let us point out that $\operatorname{Lib}(A)$ is the primitive part of $\boldsymbol{C}\langle\langle A\rangle\rangle$, then $\sum_{n \geq 0} l^{n} t^{n}$ is the Poincaré series of the enveloping algebra of the holonomy Lie algebra $\operatorname{Lib}(A)$ of $M$.

\section{Witt formula for the braid groups}

\section{Braid groups}

A braided $n$-path is a set of $n$ paths $f_{1}, \cdots, f_{n}$ in $\boldsymbol{R}^{3}$ satisfying:

i ) for any $t \in[0,1], f_{i}(t) \neq f_{j}(t)$ if $i \neq j$

ii) $f_{i}(0)=i$ for $i=1, \cdots, n$

iii) $\left\{f_{1}(1), \cdots, f_{n}(1)\right\}=\{1, \cdots, n\}$.

Two braided $n$-paths are equivalent iff it is possible to deform one into the other respecting the three above conditions throughout the deformation.

A $n$-braid is an equivalence class of braided $n$-paths.

\section{Examples.}



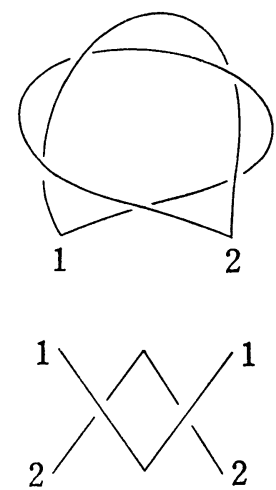
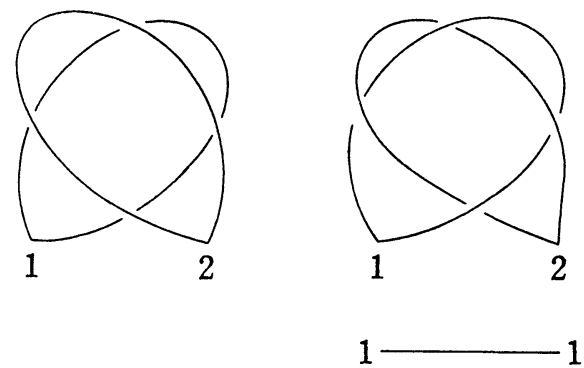

equivalent to

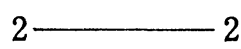

Two braids can be multiplied and we get the group $B(n)$.

The $\operatorname{map} p: B(n) \rightarrow S_{n}$, where $S_{n}$ is the symmetric group

$$
f \rightsquigarrow \sigma_{f}=\left(\begin{array}{ccc}
1 & \cdots & n \\
f_{1}(1) & \cdots & f_{n}(1)
\end{array}\right)
$$

is a homomorphism.

Ker $p:=C(n)$ is called the colored (or pure) braid group.

Let $M=C^{n} \backslash \bigcup_{1 \leqq \imath \leqq \jmath \leqq n} H_{\imath \jmath}$ where $H_{\imath \jmath}=\left\{\left(z_{1}, \cdots, z_{n}\right) \in \boldsymbol{C}^{n}\right.$ such that $\left.z_{i} \neq z_{j}\right\}$. The set of the hyperplanes $H_{\imath}$ is the complexified Coxeter arrangement of type $A_{n-1}$

$$
\boldsymbol{C}(n) \approx \pi_{1}(M ; *)
$$

\section{Cohomology of $M$}

PROPOSITION [Ar]. Let $\mathcal{A}_{n}$ be the algebra of holomorphic differential forms generated on $\boldsymbol{C}$ by $\omega^{\imath}=d \log \left(z_{i}-z_{\jmath}\right)$ for $1 \leqq i<j \leqq n$ where $z=\left(z_{1}, \cdots, z_{n}\right) \in \boldsymbol{C}^{n}$. Then $\mathcal{A}_{n} \approx H^{*}(M ; C)$ where at $\omega^{2 s}$ is associated its de Rham cohomology class $\left[\omega^{2 j}\right]$. A presentation of $\mathcal{A}_{n}$ can be given by

-the generators $\omega^{23}$ for $1 \leqq i<j \leqq n$

- the relations $\omega^{\imath \jmath} \wedge \omega^{j k}+\omega^{j k} \wedge \omega^{k \imath}+\omega^{k \imath} \wedge \omega^{2 \jmath}=0$ where $i, j, k$ are distinct and $\omega^{2 \jmath}=\omega^{j i}$.

Corollary. The Poincaré polynomial $P_{M}(t)$ is

$$
P_{M}(t)=\Pi_{k=1}^{n-1}(1+k t) .
$$

Notice that $\{1, \cdots, n-1\}$ is the set of the exponents of the Coxeter group of type $A_{n-1}$.

The proofs of these results follow from the tower of fibrations: 


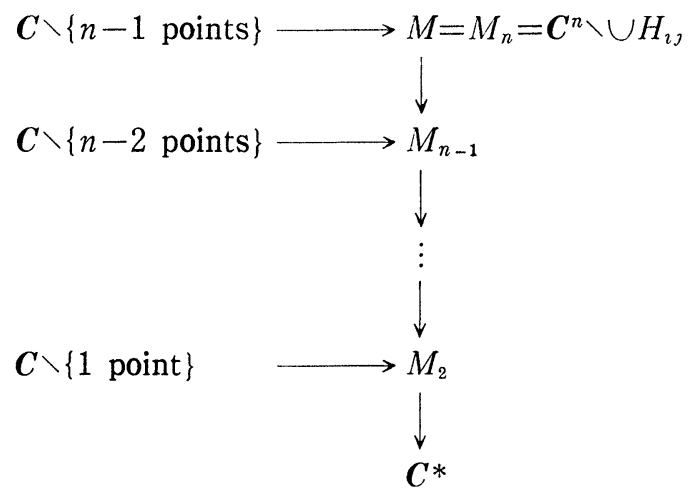

where $M_{k} \rightarrow M_{k-1}$ is the projection on the last $k-1$ factors. Moreover $M$ is an Eilenberg-MacLane space of type $K(C(n) ; 1)$.

\section{Holonomy Lie algebra of $M$.}

Let $\omega=\sum_{1 \leqq \imath<j \leqq n} A^{2 \jmath} \omega^{\imath \jmath}$ be the 1 -form on $M$ where $A^{\imath \jmath}$ is a $m \times m$ complex constant matrix and

$$
d F=\omega F
$$

The solutions are holomorphic $g l(m ; C)$-valued functions defined in open sets of $M$. Let $\gamma$ be a loop in $M$,

$$
\gamma:[0,1] \longrightarrow M
$$

and let $F_{0}$ be a solution in a neighborhood of $\gamma(0)$. By analytic continuation of $F_{0}$ along $\gamma$, we get the solution $F_{1}$ in a neighborhood of $\gamma(0)=\gamma(1)$. This solution is given by the Lappo-Danilevsky formula:

$$
F_{1}(z)=F_{0}(z) T(\gamma)
$$

where $T(\gamma)=\Sigma_{p \geqq 0} \int_{\gamma}^{p \text { times }}$

As in the preceding section, let $\nabla$ be the associated connection on the trivial bundle $\boldsymbol{C}^{m} \times M \rightarrow M$.

LEMMA. The connection $\nabla$ is flat iff:

$$
\begin{aligned}
& {\left[A^{\imath \jmath}, A^{i k}+A^{j k}\right]=0 \text { for } i, j, k \text { distinct }} \\
& {\left[A^{\imath \jmath}, A^{k \imath}\right]=0 \text { for } i, \jmath, k, l \text { distinct. }}
\end{aligned}
$$

Proof. The curvature vanishes i.e. :

$$
d \omega+\omega \wedge \omega=0
$$

iff $\omega \wedge \omega=0$ which is a consequence of the defining relations of $A_{n}$ given in the 
proposition II. 2.

Therefore the monodromy representation is given by:

$$
\begin{aligned}
& \rho: \pi_{1}(M ; *) \longrightarrow G l(m ; C) \\
& \gamma \rightsquigarrow \rho(\gamma)=\Sigma \int_{\gamma} \omega \cdots \omega
\end{aligned}
$$

Now, define $R:=C\left\langle\left\langle X_{12}, \cdots, X_{\imath \jmath}, \cdots, X_{n-1, n}\right\rangle\right\rangle / I, 1 \leqq i<j \leqq n$ where $I$ is the ideal generated by the elements:

$$
\begin{aligned}
& {\left[X_{\imath \jmath}, X_{i k}+X_{j k}\right] \text { for } i, j, k \text { distinct }} \\
& {\left[X_{\imath \jmath}, X_{k l}\right] \text { for } i, j, k, l \text { distinct. }}
\end{aligned}
$$

Let $\omega=\sum_{1 \leqq \imath<j \leqq n} \omega^{\imath \jmath} X_{\imath j} \in \mathcal{A}_{n} \otimes R$ which is called universal integrable 1-form on $M$ and the homomorphism:

$$
\begin{aligned}
& \theta: \pi_{1}(M ; *) \longrightarrow R \\
& \gamma \leadsto \sum_{k \geqq 0} \int_{\gamma} \omega^{\imath_{1} \jmath_{1}} \cdots \omega^{\imath_{k} \jmath_{k}} X_{\imath_{1} \jmath_{1}} \cdots X_{\imath_{k} \jmath_{k}}
\end{aligned}
$$

The monodromy representation $\rho$ is obtainod from $\theta$ by substituting $\boldsymbol{A}^{\imath \jmath} \in$ $g l(m ; \boldsymbol{C})$ to $X_{\imath \jmath}$.

$R$ is a Hopf algebra where the coproduct $\Delta$ is defined by $\Delta\left(X_{\imath \jmath}\right)=X_{i j} \otimes 1+$ $1 \otimes X_{\imath \jmath}$, i.e. $X_{\imath \jmath}$ is a primitive element. The primitive part of $R$, denoted $\mathcal{G}_{M}$ is called the holonomy Lie algebra of $M$.

THEOREM [K3].

$$
\Pi_{\jmath \geqq 1}\left(1-t^{\jmath}\right)^{-\varphi_{\jmath-1}(M)}=\sum_{p \geqq 0} \chi(p) t^{p}=\left(P_{M}(-t)\right)^{-1}
$$

where $\varphi_{\jmath}(M)=\operatorname{rank}$ of $\Gamma_{j} C(n) / \Gamma_{\jmath+1} C(n)$

$$
\begin{aligned}
& \sum_{p \geq 0} \chi(p) t^{p} \text { is the Poincaré series of the enveloping algebra of } \mathcal{G}_{M} \\
& P_{M}(t)=\prod_{k=1}^{n-1}(1+k t) \text { is the Poincaré polynomial of } M \text {. }
\end{aligned}
$$

In [K4], T. Kohno extends this result to the other complexified Coxeter arrangements. Notice that the left hand side equality is true for any complement of hypersurfaces.

\section{Generalized Witt formula}

Let $\mathcal{A}$ be a finite family of codimension 1 linear subspaces of $\boldsymbol{C}^{n}$ and let $M=C^{n} \backslash \bigcup_{H \in A} H$.

1. Cohomology of $M$

E. Brieskorn [B] generalized the result of Arnold as follows; let $A_{M}$ be the 
algebra of holomorphic differential forms on $M$ generated by $\omega=d \log \varphi$ where $\operatorname{ker} \varphi=H$ for $H \in \mathcal{A}$. Then there exists a natural isomorphism:

$$
\begin{aligned}
& \mathcal{A}_{M} \longrightarrow H^{*}(M ; C) \\
& \omega M \longrightarrow[\omega]
\end{aligned}
$$

Let $\mathcal{E}=\Lambda(\mathcal{A})$ be the free exterior algebra over $\mathcal{A}$. If $J=\left\{\imath_{1}, \cdots, i_{p}\right\} \subseteq$ $\{1, \cdots,|\mathcal{A}|\}$, we write $e_{J}=H_{\imath_{1}} \wedge \cdots \wedge H_{\imath_{p}}$ and $\partial e_{J}=\sum_{k=1}^{p}(-1)^{k-1} H_{\imath_{1}} \wedge \cdots \wedge \hat{H}_{\imath_{k}} \wedge$ $\cdots \wedge H_{i_{p}}$ where ${ }^{\wedge}$ means deletion. $J$ is called dependent if $\operatorname{codim}\left(H_{i_{1}} \cap \cdots \cap H_{i_{p}}\right)$ $<p$.

Let $\rho: \mathcal{E} \rightarrow \mathcal{A}_{M}$ be the algebra map which sends $H_{\imath}$ to $\omega^{2}$ for any $i=1, \cdots,|\mathcal{A}|$.

Proposition [OS]. The map $\rho: \mathcal{E} \rightarrow \mathcal{A}_{M}$ is surjective and $\operatorname{ker} \rho$ is the ideal $I$ generated by $\left\{\partial e_{J}, J\right.$ dependent $\}$.

Proposition [OS]. The Poincaré polynomial is

$$
P_{M}(t)=\Sigma_{p \geq 0}\left(\operatorname{dim} H^{p}(M)\right) t^{p}=\Sigma_{x \in L(A)} \mu(x)(-t)^{\operatorname{codim} x}
$$

where $L(A)$ is the intersection lattice ordered by reverse inclusion, $\mu(x)=\mu(0, x)$, $\mu$ being the Mobius function.

Then $\mathcal{A}_{M} \approx \mathcal{E} / I$.

Let us consider a linear order on $\mathcal{A}: H_{1}<H_{2}<\cdots . A$ set $\left\{H_{\imath_{1}}, \cdots, H_{\imath_{p}}\right\}$ is called a circuit if codim $\cap \bigcap_{j=1}^{p} H_{\imath_{j}}=p-1$ and $\operatorname{codim}\left(H_{\imath_{1}} \cap \cdots \cap \hat{H}_{\imath_{k}} \cap \cdots \cap H_{\imath_{p}}\right)=$ $p-1$ for any $k=1, \cdots, p$. Suppose $H_{\imath}<H_{\imath_{k}}$ if $\jmath<k$. Then the subset $\left\{H_{\imath_{1}}\right.$, $\left.\cdots, H_{\imath_{p-1}}\right\}$ is called a broken-circuit. We define the module $C(M)=\bigoplus_{k \geqq 0} C_{k}(M)$ where $C_{0}(M)=K$ is the ground ring and $C_{k}(M)$ is the free module with the base $\left\{H_{\imath_{1}} \wedge \cdots \wedge H_{\imath_{k}}\right\}$ such that $\left\{H_{\imath_{1}}, \cdots, H_{\imath_{k}}\right\}$ does not countain any brokencircuit.

Proposition [JT]. $\mathcal{E}=C(M) \oplus I$.

Then $\mathcal{A}_{M} \approx H^{*}(M) \approx \mathcal{E} / I \approx C(M)$.

\section{Holonomy Lie algebra of $M$}

Let $\omega=\sum \omega^{k} A_{k} \in \mathcal{A}_{M} \otimes g l(m ; C)$, the summation is taken for all $\omega^{k}=d \log \varphi_{k}$ where $\operatorname{ker} \varphi_{k}=H_{k} \in \mathcal{A}$. As above, this 1-form defines a connection $\nabla$ on the trivial bundle $\boldsymbol{C}^{m} \times M \rightarrow M$.

Notice that $d \omega=0$, then $\nabla$ is flat iff

$$
\omega \wedge \omega=0
$$

$$
\text { i. e. } \sum_{<_{k}} \omega^{\jmath} \wedge \omega^{k}\left[A^{\jmath}, A^{k}\right]=0
$$

where $[A, B]=A . B-B . A$.

The exterior product of differential forms corresponds to the cup product 
for the cohomology classes $[\omega]$ :

$$
H^{1}(M) \times H^{1}(M) \longrightarrow H^{2}(M)
$$

Let $\left\{\nu^{1}, \cdots, \nu^{p}\right\}$ be a base of $H^{2}(M)$, then:

$$
\left[\omega^{j} \wedge \omega^{k}\right]=\left[\omega^{j}\right] \cup\left[\omega^{k}\right]=\sum_{l=1}^{p} a^{j k}{ }_{l} \nu^{l}
$$

Therefore $\omega \wedge \omega=0$ iff $\sum_{\jmath_{<k}} a^{j k}{ }_{l}\left[A^{\jmath}, A^{k}\right]=0, l=1, \cdots, p$.

Let $\delta: H_{2}(M) \rightarrow H_{1}(M) \times H_{1}(M)$ be the dual morphism of the cup product morphism and let $\left\{X_{1}, \cdots, X_{q}\right\}$ be the dual base of the base $\left\{\left[\omega^{1}\right], \cdots,\left[\omega^{q}\right]\right\}$ of $H^{1}(M)$.

Consider the algebra $R=C\left\langle\left\langle X_{1}, \cdots, X_{q}\right\rangle\right\rangle / I$ where $I$ is the ideal generated by the image of $\delta$, i.e. by the elements $\sum_{\jmath_{<k}} a^{j k}{ }_{l}\left[X_{\jmath}, X_{k}\right], l=1, \cdots, p$.

Let $\omega=\Sigma_{k} \omega^{k} X^{k} \in \mathcal{A}_{M} \otimes R$ and let be the following homomorphism:

$$
\begin{aligned}
& \theta: \pi_{1}(M ; *) \longrightarrow R \\
& \gamma \rightsquigarrow \sum_{k \geq 0} \int_{\gamma} \omega^{\imath_{1}} \cdots \omega^{2_{k}} X_{\imath_{1}} \cdots X_{\imath_{k}}
\end{aligned}
$$

The monodromy representation :

$$
\rho: \pi_{1}(M ; *) \longrightarrow G l(m ; C)
$$

is again obtained from $\theta$ by substituting $A^{2} \in g l(m ; \boldsymbol{C})$ to $X_{2}$.

The holonomy Lie algebra of $M$, denoted $\mathcal{G}_{M}$, is the primitive part of $R$.

Remark. $\omega$ corresponds to the identity of $\left(H^{1}(M)\left(\otimes\left(H^{1}(M)\right)^{*}\right.\right.$ and is independent of the choice of the bases.

Proposition [K1]. $\mathcal{G}_{M}$ is isomorphic to $\operatorname{Lib}\left(X_{1}, \cdots, X_{q}\right) / \Omega$ where $\pi$ is the ideal generated by the elements $\left[\sum_{j=1}^{s} X_{\imath_{j}}, X_{\imath_{s}}\right]$ such that $\operatorname{codim} \bigcap_{j=1}^{s} H_{\imath_{j}}=2$ and $\operatorname{codim}\left(\bigcap_{j=1}^{s} H_{\imath_{j}}\right) \cap H>2$ for any $H \notin\left\{H_{\imath_{1}}, \cdots, H_{\imath_{s}}\right\}$.

\section{Some examples}

1. Let $M=C^{l} \backslash \bigcup_{l_{-1}}^{l} H_{2}$ where $H_{\imath}=\left\{z=\left(z_{1}, \cdots, z_{l}\right) \in C^{l}\right.$ such that $\left.z_{i}=0\right\}$. Then $\mathcal{G}_{M}=H^{1}(M)$ and $\operatorname{dim}_{c} \mathcal{E}_{p}\left(\mathcal{G}_{M}\right)=l$ if $p=1$ and $=0$ if $p>1$, i. e. $\varphi_{j}(M)=0$ if $j \geqq 1$. On the other hand, $P_{M}(t)=(1+t)^{l}$ and the Witt formula is satisfied.

Remark [Ao]. If $A(t)=\sum_{p \geqq 0} a_{p} t^{p}$ and $B(t)=\sum_{p \geqq 0} b_{p} t^{p}$ are two power series with real coefficients, define $A(t) \leqq B(t)$ if $a_{p} \leqq b_{p}$ for all $p \geqq 0$. Then $(1-t)^{-l} \leqq$ $\sum_{p \geqq 0} \chi(p) t^{p} \leqq(1-l t)^{-1}$ where $(1-t)^{-l}$ corresponds to the arrangement of coordinate hyperplanes of $C^{l}$ thus to the holonomy Lie algebra which is abelian and $(1-l t)^{-1}$ to $C \backslash\{l$ points $\}$ thus to the holonomy Lie algebra which is free.

2. This arrangement denoted $\mathfrak{X}_{1},[F R]$, does not satisfy the Witt formula. 


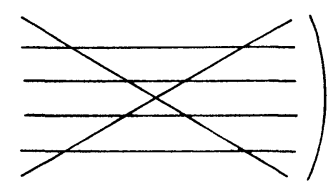

\section{Fiber-type arrangements}

Definition. The arrangement $\mathcal{A}$ in $\boldsymbol{C}^{n}$ is fiber-type if there is a tower of bundle maps :

$$
M=M_{n} \stackrel{p_{n}}{\rightarrow} M_{n-1} \longrightarrow \cdots \longrightarrow M_{2} \stackrel{p_{2}}{\longrightarrow} M_{1}=C^{*}
$$

such that for each $k, 2 \leqq k \leqq n$ :

(i) $M_{k}$ is the complement of an arrangement in $C^{k}$

(ii) $p_{k}$ is the restriction of a linear map $\boldsymbol{C}^{k} \rightarrow C^{k-1}$

(iii) the fiber $F_{k}$ of $p_{k}$ is a copy of $C \backslash\{$ finite points $\}$.

These numbers $\left\{a_{1}, \cdots, a_{n}\right\}$ of points removed of $C$ in each fiber are called exponents of $\mathcal{A}$ and

$$
P_{M}(t)=\Pi_{2=1}^{n}\left(1+a_{i} t\right)
$$

Notice that the complexified Coxeter arrangements of type $A_{n-1}$ are fibertype with exponents $\{1,2, \cdots, n-1\}$.

TheOREM [J]. Let the bundle map $p_{n}: M \rightarrow M_{n-1}=N$, then the natural map:

$$
\varphi: \operatorname{Lib}\left(A_{1}\right) \oplus \mathcal{G}_{N} \longrightarrow \mathcal{G}_{M}
$$

where $A_{1}=\left\{X_{1}, \cdots, X_{a_{1}}\right\}$ is a graded linear isomorphism.

COROLlaRY [J]. Let $\mathcal{A}$ be a fiber-type arrangement of $\boldsymbol{C}^{n}$; then there exists a graded linear isomorphism:

$$
\oplus_{i=1}^{n} \operatorname{Lib}\left(A_{\imath}\right) \longrightarrow \mathcal{G}_{M}
$$

where $\left|A_{\imath}\right|=a_{\imath}$ for $\imath=1, \cdots, n$.

COROLLARY [FR], [J], [K]. The Witt formula is satisfied for the fiber-type arrangements.

Examples. The arrangement denoted $J_{2}$ is not fiber-type does not satisfy the Witt formula $[\mathrm{FR}]$ and is free, $P_{M}(t)=(1+t)(1+3 t)^{2}$. 


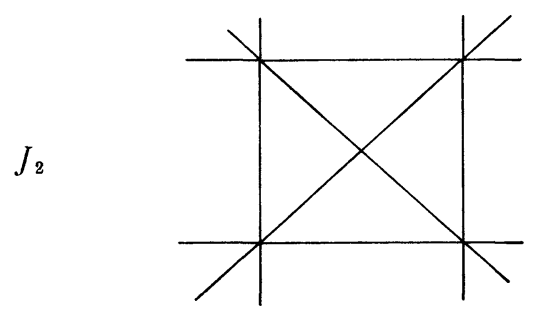

The arrangement denoted $\mathfrak{X}_{2}$ is not free, not fiber-type and satisfies the Witt formula, $P_{M}(t)=(1+t)(1+3 t)^{2}$.

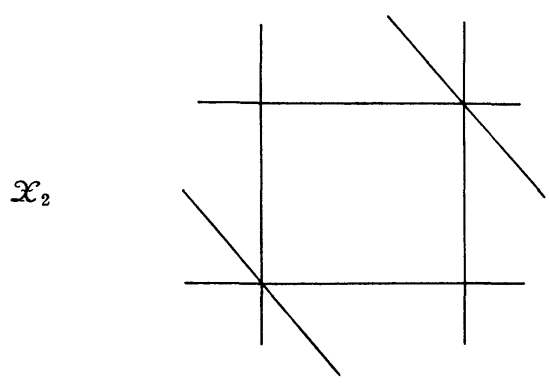

\section{Aomoto's complex}

\section{A resolution of $Q$}

Let $M$ be the complement of an arrangement $A$ of $C^{n}$. Let $\left\{\omega^{1}, \cdots, \omega^{\mid A^{A}}\right\}$ be a base of $H^{1}(M ; \boldsymbol{Q})$, e.g. $\omega^{2}=d \log \varphi_{i}$ where $\operatorname{ker} \varphi_{i}=H_{i} \in \mathcal{A}$ and let $\left\{X_{1}, \cdots\right.$, $\left.X_{|A|}\right\}$ be the dual base of $H_{1}(M ; \boldsymbol{Q})$. Let

$$
R^{k}=\mathcal{E}\left(\mathcal{G}_{M}\right) \otimes_{\boldsymbol{Q}} H^{k}(M ; \boldsymbol{Q}), \quad k \geqq 0
$$

and define the $\mathcal{E}\left(\mathcal{G}_{M}\right)$-modules morphism:

$$
\begin{aligned}
& \delta: R^{k-1} \longrightarrow R^{k} \\
& 1 \otimes \varphi . \rightsquigarrow \sum_{\imath-1} X_{i} \otimes\left(\omega^{2} \cup \varphi\right), \quad \varphi \in H^{k-1}(M ; \boldsymbol{Q})
\end{aligned}
$$

Let $R_{k}=\operatorname{Hom}_{\mathcal{E}\left(g_{M}\right)}\left(R^{k}, \mathcal{E}\left(\mathcal{G}_{M}\right)\right), k \geqq 0$ and

$$
\partial_{k}: R_{k} \longrightarrow R_{k-1}
$$

the dual morphism of $\delta^{k}$.

Then $\left(R ., \partial\right.$.) is a complex and the differential $\partial_{k}, k \geqq 0$, does not depend of the choice of the bases. This complex was introduced by K. Aomoto [Ao].

Proposition $[\mathrm{K} 3,4]$. If the complex $(R ., \partial$.$) satisfies H_{j}(R)=$.0 for $j>0$ then:

i) there is a resolution of $\boldsymbol{Q}$ as a $\mathcal{E}\left(\mathcal{G}_{M}\right)$-module: 


$$
0 \longrightarrow R_{n} \stackrel{\partial_{n}}{\longrightarrow} R_{n-1} \longrightarrow \cdots \longrightarrow R_{1} \stackrel{\partial_{1}}{\longrightarrow} R_{0} \stackrel{\varepsilon}{\longrightarrow} \boldsymbol{Q} \longrightarrow 0
$$

where $\operatorname{rank} R_{\jmath}=\operatorname{dim} H^{\jmath}(M ; \boldsymbol{Q})$ and $\varepsilon$ is the augmentation morphism

ii) $M$ satisfies the Witt formula

$$
\Pi_{\jmath \geq 1}\left(1-t^{\jmath}\right)^{-\varphi_{\jmath-1}(M)}=\sum_{p \geqq 0} \chi(p) t^{p}=\left(P_{M}(-t)\right)^{-1} .
$$

The following lemma (which is due to T. Kohno [K4]) is used to prove the next proposition.

Let $\mathcal{B}$ be a subset of $\mathcal{A}$. by:

LEMMA. Let $d^{\mathscr{G}}: \mathcal{E}\left(\mathscr{G}_{M}\right)^{\mathscr{B}} \rightarrow \mathcal{E}\left(\mathscr{G}_{M}\right)$ be the (right) $\mathcal{E}\left(\mathscr{G}_{M}\right)$-module morphism defined

$$
d^{\mathscr{B}}(u)=\Sigma_{\imath} u_{\imath} X_{\imath} \quad \text { for } u=\left(u_{\imath}\right) \in \mathcal{E}\left(\mathcal{Q}_{N}\right)^{\mathscr{B}} \text { and } H_{i} \in \mathscr{B} .
$$

Let $C$ be the degree 1 part of $\operatorname{Ker} d^{\mathscr{B}}$ and denote $\operatorname{Lib}(A)_{\mathscr{B}}$ the Lie subalgebra of $\operatorname{Lib}(A)$ generated by the $X_{\imath}$ such that $H_{i} \in \mathscr{B}$. Let $\varphi: \mathscr{I}^{(2)} \cap \operatorname{Lib}(A)_{\mathscr{B}} \rightarrow C V$ be the linear map defined by:

$$
\varphi(r)=\left(\partial r / \partial X_{\imath}\right) \text { for } i \text { such that } H_{i} \in \mathscr{B} .
$$

Then $\operatorname{Ker} d^{\mathscr{B}}$ is generated by $\mathscr{V}$ as a $\mathcal{E}\left(\mathcal{G}_{M}\right)$-module. Moreover $\varphi$ is an isomorphism of vector-spaces.

PROPOSITION. $\partial_{n}$ is injectıve.

Proof. Define a linear order $<$ on the set $A$ of hyperplanes:

$$
H_{1}<H_{2}<\cdots<H_{|\mathcal{A}|}
$$

and denote $H:=H_{|\mathcal{A}|}$. Let us recall (II 2) that $C(M)=\oplus_{k \geq 0} C_{k}(M)$ where $C_{0}(M)$ $=\boldsymbol{Q}$ and $C_{k}(M)$ is the $\boldsymbol{Q}$-linear space with the base $\left\{H_{\imath_{1}} \wedge \cdots \wedge H_{\imath_{k}}\right\}$ such that $\left\{H_{\imath_{1}}, \cdots, H_{\imath k}\right\}$ does not countain any broken-circuit. If we assume $i_{1}<i_{2}<\cdots<\imath_{k}$, then this base is called $B C$-standard. Then the $B C$-standard base of $C_{n}(M)$ is $\left\{\varphi \wedge H\right.$ such that $\varphi$ belongs to the $B C$-standard base of $\left.C_{n-1}(M)\right\},\{1 \otimes(\varphi \wedge H)$ for all such $\varphi\}$ is a base of $R^{n}$ and by duality $\left\{1 \otimes(\varphi \wedge H)^{*}\right.$ for all such $\left.\varphi\right\}$ is the dual base of $R_{n}$. Therefore for $\varphi$ and $\phi$ in the $B C$-standard base of $C_{n-1}(M)$ :

and

$$
\partial_{n}\left(1 \otimes(\varphi \wedge H)^{*}\right)(1 \otimes \phi)=\left(1 \otimes(\varphi \wedge H)^{*}\right)\left(\Sigma_{H_{i} \in A} X_{i} \otimes\left(H_{\imath} \wedge \psi\right)\right)
$$

$$
\begin{aligned}
& \partial_{n}\left(1 \otimes(\varphi \wedge H)^{*}\right)(1 \otimes \varphi)= \pm X+\Sigma_{\alpha}\left( \pm X_{\alpha}\right) \quad \text { where } X_{\alpha} \neq X \\
& \partial_{n}\left(1 \otimes(\varphi \wedge H)^{*}(1 \otimes \psi)=\Sigma_{\beta}\left( \pm X_{\beta}\right) \quad \text { where } X_{\beta} \neq X \text { and } \phi \neq \varphi .\right.
\end{aligned}
$$

Let $\left.\Sigma_{\varphi} f_{\varphi} \partial_{n}\left(1 \otimes(\varphi \wedge H)^{*}\right)\right)=0$ where $f_{\varphi} \in \mathcal{E}\left(\mathcal{G}_{M}\right)$ and the sum is over $\varphi$ in the $B C$ standard base of $C_{n-1}(M)$. Suppose there exists $f_{\psi} \neq 0$, then: 


$$
\sum f_{\varphi} \partial_{n}\left(1 \otimes(\varphi \wedge H)^{*}\right)(1 \otimes \psi)=f_{\psi}\left( \pm X+\Sigma_{\alpha} \pm\left(X_{\alpha}\right)\right)+\Sigma_{\varphi \neq \psi} f_{\varphi}\left(\Sigma_{\beta}\left( \pm X_{\beta}\right)\right)
$$

where $X_{\alpha}$ and $X_{\beta} \neq X$. Let us denote $f_{\psi}={f^{\prime}}^{\prime} \cdot X_{\psi}$, then $f_{\psi} \cdot X={f^{\prime}}_{{ }^{\prime}} \cdot X_{\psi} \cdot X$. By the lemma, there exists $\nu$ such that $f_{\nu}=-f^{\prime}{ }_{\psi} \cdot X$.

$$
\begin{aligned}
f_{\nu} \partial_{n}\left(1 \otimes(\nu \wedge H)^{*}\right)(1 \otimes \nu) & =f_{\nu} \cdot X+f_{\nu} \Sigma_{\gamma}\left( \pm X_{\gamma}\right) \\
& =-f^{\prime}{ }_{{ }^{\prime}} \cdot X^{2}-f^{\prime}{ }_{\phi} X \Sigma_{\gamma}\left( \pm X_{\gamma}\right) \\
f_{\varphi} \partial_{n}\left(1 \otimes(\varphi \wedge H)^{*}\right)(1 \otimes \nu) & =f_{\mu} \Sigma_{a}\left( \pm X_{\alpha}\right) .
\end{aligned}
$$

Using lemma, we get:

$$
\Sigma_{\varphi} f_{\varphi} \partial_{n}\left(1 \otimes(\varphi \wedge H)^{*}\right)(1 \otimes \nu) \pm 0
$$

and the result follows.

THEOREM [K4]. The complex $(R,, \partial$, ) associated with the Coxeter arrangements of type $A_{l}, C_{l}, D_{l}$ are acyclic.

N.B.: The main difficulty of the proof is the injectivity of $\partial_{n}$.

\section{2. Acyclicity of the Aomoto's complex}

Suppose there exists a structure of graded algebra on $H^{*}(M ; \boldsymbol{Q})$ and let $K . H^{*}(M ; \boldsymbol{Q})$ be the associated decreasing filtration :

$$
K_{-p} H^{*}(M ; \boldsymbol{Q})=\left\{x \in H^{*}(M ; \boldsymbol{Q}), \text { such that } \operatorname{deg} x \leqq p\right\}
$$

Let :

$$
G r_{-p} H^{*}(M ; \boldsymbol{Q})=K_{-p} H^{*}(M ; \boldsymbol{Q}) / K_{-p+1} H^{*}(M ; \boldsymbol{Q})
$$

The filtration $K$. on $H^{*}(M ; \boldsymbol{Q})$ induces a filtration on the complex $(R ., \partial$.) by

$$
K_{-p} R_{k}=\operatorname{Hom}_{\mathcal{E}\left(\underline{g}_{M}\right)}\left(\mathcal{E}\left(\mathcal{G}_{M}\right) \otimes_{Q} K_{-p} H *(M ; \boldsymbol{Q}) ; \mathcal{E}\left(\mathcal{G}_{\boldsymbol{M}}\right)\right)
$$

The natural projection map:

$$
\pi_{p}: K_{-p} H^{k}(M ; \boldsymbol{Q}) \longrightarrow K_{-p+1} H^{k}(M ; \boldsymbol{Q})
$$

induces an injective morphism of $\mathcal{E}\left(\mathscr{Q}_{M}\right)$-modules :

$$
K_{-p+1} R_{k} \longrightarrow K_{-p} R_{k}
$$

LEMMA. $\partial$ is compatible with the filtration, i.e.

$$
\partial_{k}\left(K_{-p} R_{k}\right) \subseteq K_{-p} R_{k-1}
$$

Proof. Consider the map:

$$
\begin{aligned}
& \delta^{k}: \mathcal{E}\left(\mathcal{G}_{M}\right) \otimes_{Q} K_{-p} H^{k-1}(M ; \boldsymbol{Q}) \longrightarrow \mathcal{E}\left(\mathcal{G}_{M}\right) \otimes_{Q} K_{-p} H^{k}(M ; \boldsymbol{Q}) \\
& 1 \otimes \varphi \rightsquigarrow \hookrightarrow \Sigma_{\imath-1} X_{i} \otimes\left(\left[\omega^{i}\right] \cup \varphi\right)
\end{aligned}
$$


and the commutative diagram:

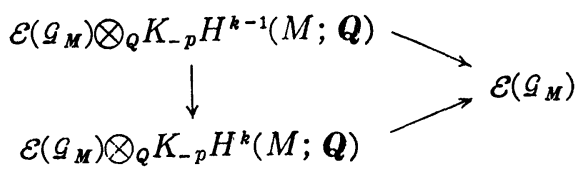

The result follows.

Thus we obtain a structure of filtered complex on $(R ., \partial$.$) .$

Consider the spectral sequence of this filtered complex. We put:

$$
\begin{aligned}
& Z_{r}^{p q}=\left\{x \in K_{p} R_{-p-q}, \partial x \in K_{p+r} R_{-p-q+1}\right\} \\
& B_{r}^{p q}=\left\{x \in K_{p} R_{-p-q}, \text { there exists } y \in K_{-p-r} R_{-p-q-1} \text { such that } x=\partial y\right\} \\
& E_{r}^{p q}=Z_{r}^{p q} /\left(B_{r-1}^{p q}+Z^{p+1, q-1}{ }_{r-1}\right) \\
& G r_{p} R_{-p-q}=K_{p} R_{-p-q} / K_{p+1} R_{-p-q}
\end{aligned}
$$

Then $E^{p q_{0}}=G r_{r} R_{-p-q} \approx \operatorname{Hom}_{\mathcal{E}\left(G_{M}\right)}\left(\mathcal{E}\left(\mathscr{G}_{M}\right) \otimes_{Q} G r_{p} H^{-p-q}(M ; \boldsymbol{Q}), \mathcal{E}\left(\mathcal{G}_{M}\right)\right)$.

Proposition. Suppose that $E^{p q}{ }_{1}=0$ of $p+q \neq 0$. Then the complex $(R ., \partial$. $)$ is acyclic, i.e. $H_{j}(R)=$.0 for $j>0$.

Proof. The differential $d_{1}: E^{p q}{ }_{1} \rightarrow E^{p+1, q}{ }_{1}$ is the zero map. Hence we have $E^{p q}{ }_{2}=E^{p q}{ }_{1}$. By induction, we prove that the differential:

$$
d_{r}: E_{r}^{p q} \longrightarrow E^{p+r, q-r+1} r
$$

is the zero map for $r \geqq 1$. Then

$$
E^{p q}{ }_{1}=E^{p q}{ }_{2}=\cdots=E^{p q}
$$

Since $E^{p q}=K_{p} H_{-p-q}(R.) / K_{p+1} H_{-p-q}(R$.$) , the result follows.$

COROLLARY. If $E^{p q}{ }_{1}=0$ for $p+q \neq 0$, then $M$ satisfies the Witt formula.

\section{3. Filtration of the Aomoto's complex}

Let us consider a chain of $L(\mathcal{A})$ of lenght $r \leqq r(L(\mathcal{A}))$ such that:

$$
0=x_{0}<x_{1}<\cdots<x_{r-1}<x_{r}=1
$$

Let $\mathcal{A}=\bigcup_{i=1}^{r} \mathcal{A}_{2}$ be the disjoint union where $\bigcup_{j=1}^{p} \mathcal{A}_{j}=\left\{H \in \mathcal{A}, H \$ x_{r-p}\right\}$. Let us define a linear order $\prec$ on $\mathcal{A}$ such that $H_{i}<H$, if $H_{i} \in \mathcal{A}_{\imath}, H_{j} \in \mathcal{A}$, and $\imath<j$. We begin to define a decreasing filtration on the algebra $\mathcal{E}: K_{-p} \mathcal{E}=\left\{H_{\imath_{1}} \wedge \cdots \wedge H_{\imath_{q}}\right.$, such that there exists $\jmath \in\{1, \cdots, q\}$ where $\left.H_{r_{j}} \in \mathcal{A}_{r-p+1} \cup \cdots \cup \mathcal{A}_{r}\right\}$.

Therefore : $\quad \cdots \mathcal{E}=K_{-r} \mathcal{E} \supset K_{-r+1} \mathcal{E} \supset \cdots \supset K_{-1} \mathcal{E} \supset K_{0} \mathcal{E}=\boldsymbol{Q}=\cdots$ 
and

$$
K_{-p} \mathcal{E} \wedge K_{-q} \mathcal{E} \subset K_{-(p+q)} \mathcal{E}
$$

Then $\mathcal{E}$ is a filtered algebra with the following gradation:

$$
\begin{aligned}
G r_{-p} \mathcal{E}= & K_{-p} \mathcal{E} / K_{-p+1} \mathcal{E} \\
= & \left\{H_{\imath_{1}} \wedge \cdots \wedge H_{\imath_{q}} \text { such that there exists } j \in\{1, \cdots, q\}\right. \\
& \text { where } H_{\imath_{j}} \in \mathcal{A}_{r-p+1} \text { and } H_{\imath_{k}} \in \mathcal{A}_{1} \cup \cdots \cup \mathcal{A}_{r-p+1} \text { for } \\
& k=1, \cdots, q\} .
\end{aligned}
$$

In fact, $K_{-p} \mathcal{E}=\oplus_{q \geq 0}\left(K_{-p} \mathcal{E}\right)_{q}$ where $\left(K_{-p} \mathcal{E}\right)_{q}$ is the $\boldsymbol{Q}$-vector space with the base $H_{\imath_{1}} \wedge \cdots \wedge H_{\imath_{q}}$ such that $H_{\imath_{1}} \prec \cdots \prec H_{\imath_{q}}$ and $H_{\imath_{q}} \in \mathcal{A}_{r-p+1} \cup \cdots \cup \mathcal{A}_{r}$ and $\left(G r_{-p} \mathcal{E}\right)_{q}$ is the $\boldsymbol{Q}$-vector space with the base $H_{\imath_{1}} \wedge \cdots \wedge H_{\imath_{q}}$ such that $H_{\imath_{1}} \prec \cdots \prec H_{\imath_{q}}$ and $H_{\imath_{q}} \in \mathcal{A}_{r-p+1}$.

Let us recall that $\mathcal{E}=C(M) \oplus I$, then $C(M) \approx \mathcal{E} / I$.

Proposition. The above filtration $K$. on $\mathcal{E}$ induces a decreasing filtration $K$. on $C(M)$ (as algebra).

Proof. Let $a=\bigwedge_{k=1}^{s} H_{\imath_{k}}$ and $b=\bigwedge_{k=1}^{t} H_{\jmath_{k}}$ be two elements of the standard $B C$-base of $K_{-p} C(M)$ (resp. $\left.K_{-q} C(M)\right)$; then $a \in K_{-p} \mathcal{E}$ and $b \in K_{-q} \mathcal{E}$, then $a \wedge b$ $\in K_{-(p+q)} \mathcal{E}$. Moreover $a \wedge b=c+d$ where $c \in C(M)$ and $d \in l$. For simplicity, we denote $a=\bigwedge_{k=1}^{s} H_{k}$ and $b=\bigwedge_{k=s+1}^{s+t} H_{k}$. Suppose $\left\{H_{\imath_{1}}, \cdots, H_{\imath_{l}}\right\}$ is a brokencircuit included in $\left\{H_{\imath}, \imath=1, \cdots, s\right\} \cup\left\{H_{\imath}, \imath=s+1, \cdots, s+t\right\}$. Then there exists $H_{\imath_{l+1}} \succ H_{\imath}$ for any $\imath=1, \cdots, s+t$ such that $\left\{H_{\imath_{1}}, \cdots, H_{\imath_{l}}, H_{\imath_{l+1}}\right\}$ is a circuit. Therefore $H_{\imath_{j}} \in \mathcal{A}_{k}$, and $H_{\imath_{l+1}} \in \mathcal{A}_{k_{l+1}}$ where $k_{l+1} \geqq k_{\jmath}$ for any $\jmath=1, \cdots, l$. Repeat ing this operation, we finally get a sum of terms without countaining any broken-circuit, i.e. a sum of terms which belong to the $B C$-base and which is the element $c$. Moreover $c \in K_{-(p+q)} C(M)$ and $C(M)$ is a filtered algebra.

The main application of this result is the following and the proof is straighforward:

Proposition. Let $A$ be a fiber-type arrangement of $C^{l}$ and $K$. the filtration on $C(M)$ associated with a maximal modular chain of $L(\mathcal{A})$. Then $\left(G r_{-p} C(M)\right)_{q}$ is the $\boldsymbol{Q}$-vector space with the standard $B C$-base $H_{\imath_{1}} \wedge \cdots \wedge H_{\imath_{q}}$ such that $H_{\imath_{q}} \in$ $\mathcal{A}_{l-p+1}$ and each $H_{\imath_{k}} \in \mathcal{A}_{\jmath_{k}}$ where $k=1, \cdots, q-1$ and $\jmath_{k}<l-p+1, \jmath_{k}$ pairwise distinct.

COROLLARY. The spectral sequence of the associated filtered complex (R.o.) satısfies $E^{p q}=0$ for $p+q \neq 0$ and this complex is acyclic.

It is another way to prove that a fiber-type arrangement satisfies the LCS property, i.e. the generalized Witt formula $[\mathrm{FR}],[\mathrm{J}],[\mathrm{K}]$. 


\section{REFERENCES}

[Ao] Аомото, K., Poincaré series of the holonomy Lie algebra attached to a configuration of lines. Preprint, Nagoya.

[Ar] ARnold, V.I., The cohomology ring of the colored braid group, Mat. Zametki (1969), 227-231.

[B] Brieskorn, E., "Sur les groupes de tresses", sémınaire Bourbaki, $24^{\text {ème }}$ année, 1971-72, Springer Lecture Notes 317.

[Ca] CARTier, P., "Jacobiennes généralisées, monodromie unıpotente et intégrales itérées", séminaire Bourbaki, 40 ${ }^{\mathrm{ème}}$ année, 1987-88.

[Ch] Chen, K. T., Iterated path integrals, Bull. Amer. Math. Soc. 83 (1977), 831-879.

[F] FALK, M., The minimal models of the complement of an arrangement of hyperplanes, Trans. Amer. Math. Soc., 309 (1988), 543-556.

[FR1] FALK, M., RANDELl, R., The lower central series of a fiber-type arrangement, Invent. Math. 82 (1985), 77-88.

[FR2] FALK, M., RANDELL, R., On the homotopy theory of arrangements, in "Complex Analytic Singularities" Adv. Studies in Pure Math., North Holland, Amsterdam, (1986), 101-124.

[H] HaIN, R., On a generalization of Hilbert's $21^{\text {st }}$ problem, Ann. Scient. E. N.S., 19 (1986), 609-627.

[J] JAMBU, M., Fiber-type arrangements and factorization properties, Advances in Math. 80 (1990), 1-21.

[JT] Jambu, M., Terao, H., Arrangements of hyperplanes and broken-circuits, Contemporary Math., 90 (A. M.S.) (1990), 147-162.

[K1] Kohno, T., On the holonomy Lie algebra and the nilpotent completion of the fundamental group of complement of hyperplanes, Nagoya Math. J. 92 (1985), 21-37.

[K2] Koнno, T., Differential forms and the fundamental group of the complement of hyperplanes, Proc. Pure Math. Amer. Math. Soc. (1983) 655-662.

[K3] Koнno, T., Série de Poincaré-Koszul associée aux groupes de tresses pures, Invent. Math. 82 (1985), 57-75.

[K4] Konno, T., Poincaré series of the Malcev completion of generalized pure braid groups, Preprint.

[OS] ORlik, P., Solomon, L., Combinatorics and topology of complements of hyperplanes, Invent. Math. 56 (1980), 167-189.

[S] Sullivan, D., Infinitesimal computations in topology, Publ. Math. IHES, 47 (1977), 269-331.

Unité de RECHERChe ASSOCIÉE AU CNRS N ${ }^{\circ} 758$

Département de Mathématiques et d'INFormatique

UNIVERSité de NANTES

2 RUE DE LA Houssiniere

44072 Nantes Cedex 03, France 